Bull. Austral. Math. Soc.

VoL. 42 (1990) [191-200]

\title{
SUMS OF DEFICIENCIES OF ALGEBROID FUNCTIONS
}

\section{LiAn-ZhoNg YaNG}

Let $f(z)$ be an $n$-valued algebroid function of finite lower order. In the present paper, we give a spread relation of $f(z)$ and some applications of the spread relation.

\section{INTRODUCTION}

Let $f(z)$ be an $n$-valued algebroid function of finite lower order $\mu$, defined by an irreducible equation

$$
A_{0} f^{n}+A_{1} f^{n-1}+\cdots+A_{n-1} f+A_{n}=0
$$

where $A_{0}, A_{1}, \cdots, A_{n}$ are entire functions without common zeros.

Fix a sequence $\left(r_{j}\right)$ of Pólya peaks of order $\mu$ of $f(z)$ (or $T(r, f)$ ). Let $f_{j}(z)$ be the $j$ th determination of $f(z)$ and $\Lambda(r)$ a positive function with

$$
\Lambda(r)=o(T(r, f)), \quad r \rightarrow \infty .
$$

Define the sets of arguments $E_{\Lambda}^{\prime}(r, a) \subset(-\pi, \pi]$ by

$$
\begin{aligned}
E_{\Lambda}^{\prime}(r, a) & =\left\{\theta: \min _{1 \leqslant j \leqslant n}\left|f_{j}\left(r e^{i \theta}\right)-a\right|<e^{\Lambda(r)}, a \neq \infty\right\}, \\
E_{\Lambda}^{\prime}(r, \infty) & =\left\{\theta: \max _{1 \leqslant j \leqslant n}\left|f_{j}\left(r e^{i \theta}\right)\right|>e^{\Lambda(r)}\right\}, \\
\sigma_{\Lambda}^{\prime}(a) & =\liminf _{j \rightarrow \infty} \operatorname{meas} E_{\Lambda}^{\prime}\left(r_{j}, a\right) \\
\sigma^{\prime}(a) & =\inf _{\Lambda} \sigma_{\Lambda}^{\prime}(a)
\end{aligned}
$$

and let

where the infimum is taken over all functions $\Lambda(r)$ satisfying (1.2). Niino ([5]) proved the following spread relation

$$
\sigma^{\prime}(a) \geqslant \min \left\{2 \pi, \frac{4}{\mu} \arcsin \sqrt{\frac{\delta(a, f)}{2}}\right\} .
$$

Received 9 October 1989

This research was partially supported by a grant from NSF of P.R. China.

Copyright Clearance Centre, Inc. Serial-fee code: 0004-9729/90 \$A2.00+0.00. 
Now we assume that

$$
\begin{aligned}
\|A(z)\| & =\left(\left|A_{0}\right|^{2}+\left|A_{1}\right|^{2}+\cdots+\left|A_{n}\right|^{2}\right)^{1 / 2}, \\
\|a\| & = \begin{cases}\left(|a|^{2 n}+|a|^{2 n-2}+\cdots+|a|^{2}+1\right)^{1 / 2}, & a \neq \infty \\
1, & a=\infty,\end{cases} \\
F(z, a) & = \begin{cases}A_{0} a^{n}+A_{1} a^{n-1}+\cdots+A_{n-1} a+A_{n}, & a \neq \infty \\
A_{0}, & a=\infty,\end{cases} \\
m(r, a, A) & =\frac{1}{2 \pi} \int_{0}^{2 \pi} \log \left|\frac{\|A\| \cdot\|a\|}{F(z, a)}\right| d \theta, z=r e^{i \theta}, \\
\mu(r, A) & =\frac{1}{2 n \pi} \int_{0}^{2 \pi} \log \max _{0 \leqslant j \leqslant n}\left|A_{j}\left(r e^{i \theta}\right)\right| d \theta .
\end{aligned}
$$

Set

$$
T(r, a, A)=m(r, a, A)+N(r, 0, F(z, a))
$$

by Jensen's formula, we have

$$
T(r, a, A)=\frac{1}{2 \pi} \int_{0}^{2 \pi} \log (\|A\| \cdot\|a\|) d \theta+O(1) .
$$

Since

$$
\max _{0 \leqslant j \leqslant n}\left|A_{j}(z)\right| \leqslant\|A(z)\| \leqslant(n+1)^{1 / 2} \max _{0 \leqslant j \leqslant n}\left|A_{j}(z)\right|,
$$

we have

$$
|T(r, a, A)-n \mu(r, A)|=O(1) \text {. }
$$

By using Valiron's result ([6]), we get

$$
\begin{gathered}
|T(r, a, A)-n T(r, f)|=O(1), \\
\text { so that } \quad \delta(a, f)=1-\underset{r \rightarrow \infty}{\limsup } \frac{N(r, 0, F(z, a))}{T(r, a, A)}
\end{gathered}
$$

With these notations, we define the sets of arguments $E_{\Lambda}(r, a) \subset(-\pi, \pi]$ by

and let

$$
E_{\Lambda}(r, a)=\left\{\theta: \frac{\|A\| \cdot\|a\|}{|F(z, a)|}>e^{\Lambda(r)}, \quad z=r e^{i \theta}\right\}
$$

$$
\begin{aligned}
\sigma_{\Lambda}(a) & =\liminf _{j \rightarrow \infty} \text { meas } E_{\Lambda}\left(r_{j}, a\right) \\
\sigma(a) & =\inf _{\Lambda} \sigma_{\Lambda}(a)
\end{aligned}
$$

where the infimum is taken over all functions satisfying (1.2).

In the present paper, we prove a spread relation analogous to (1.3) with the spread $\sigma^{\prime}(a)$ replaced by $\sigma(a)$ and give some applications of the spread relation. 


\section{SPREAd ReLations}

In the following statements the notations of the introduction are taken for granted. For a complex number $a$, we set

$$
\begin{gathered}
m^{*}(z, a)=\sup _{E} \frac{1}{2 \pi} \int_{E} \log \frac{\|A\| \cdot\|a\|}{|F(\xi, a)|} d \omega, \quad \xi=r e^{i \omega}, \\
\left(z=r e^{i \theta}, 0<r<\infty, 0 \leqslant \theta \leqslant \pi\right)
\end{gathered}
$$

where the supremum is taken over all measurable sets $E \subset(-\pi, \pi]$ of Lebesgue measure $2 \theta$, and

$$
T^{*}(z)=T^{*}(z, a)=m^{*}(z, a)+N(r, 0, F(z, a)) .
$$

The function $T^{*}(z)$ is defined on the set

$$
H_{1}=\{z: \operatorname{Im} z \geqslant 0, \quad z \neq 0\} .
$$

It follows from the definition of this function that for arbitrary $r$ such that $0<r<\infty$ and a complex number $a$ we have

$$
\begin{aligned}
& \sup T^{*}\left(r e^{i \theta}\right)=T(r, a, A), \\
& T^{*}(r)=N(r, 0, F(z, a)) .
\end{aligned}
$$

LEMma 2.1. $T^{*}(z)$ is subharmonic in the half plane $\operatorname{Im} z>0$ and is continuous in $H_{1}$.

Proof: By a result of Goldberg ([3]), we know that $\log \|A\|$ is subharmonic so that $\log (\|A\| \cdot\|a\|)$ is subharmonic. Since $F(z, a)$ is an entire function, we have $\log |F(z, a)|$ is a subharmonic function. By the Theorem $\mathrm{A}^{\prime}$ in [2], Lemma 2.1 follows.

THEOREM 2.1. Let $f(z)$ be an $n$-valued algebroid function of lower order $\mu(0<\mu<\infty)$, defined by the equation (1.1); then

$$
\sigma(a) \geqslant \min \left\{2 \pi, \frac{4}{\mu} \arcsin \sqrt{\frac{\delta(a, f)}{2}}\right\},
$$

where $a$ is a deficient value of $f(z)$.

Proof: We consider the following two cases.

(1) $4 \arcsin \sqrt{(\delta(a, f) / 2)} / \mu<2 \pi$.

To deduce inequality (2.3) we should use Lemma 2.1 and the proof of (1.4) in [1]; let us outline the method of the proof of inequality (2.3) (for details see the proof of relation (1.4) in $[1, \mathrm{p} .429-434]$. 
We set

$$
\gamma=\frac{2}{\pi \mu} \arcsin \sqrt{\frac{\delta(a, f)}{2}} .
$$

The following inequality is fulfilled for the function

$$
v(z)= \begin{cases}0, & z=0 \\ T^{*}\left(z^{\gamma}\right), & z=r e^{i \theta}, 0<r<\infty, 0 \leqslant \theta \leqslant \pi\end{cases}
$$

which is subharmonic in the half plane $\operatorname{Im} z>0$ (see Lemma 2.1):

$$
v\left(r e^{i \theta}\right) \leqslant \int_{-R}^{R} v(t) A(t, r, \theta, R) d t+\int_{0}^{\pi} v\left(R e^{i \varphi}\right) B(\varphi, r, \theta, R) d \varphi
$$

where $A$ and $B$ are kernels (see $[1, p .430]$ ).

We use the estimates $B(\varphi, r, \theta, R)<32(r / R),(0<\theta<\pi, 0<\varphi<\pi, 0<r<R / 2)$ and $A(t, r, \theta, R) \leqslant P(t, r, \pi-\theta), A(-t, r, \theta, R) \leqslant P(t, r, \theta)$, where

$$
P(t, r, \theta)=\frac{1}{\pi} \frac{r \sin \theta}{t^{2}+2 r t \cos \theta+r^{2}} \text {. }
$$

Taking into account properties (2.1) and (2.2) of the function $T^{*}(z)$, we get

$$
\begin{aligned}
v\left(r e^{i \theta}\right) \leqslant & \int_{0}^{R} N\left(t^{\gamma}, 0, F(z, a)\right) P(t, r, \pi-\theta) d t \\
& +\int_{0}^{R} T\left(t^{\gamma}, a, A\right) P(t, r, \theta) d t+32(r / R) T\left(R^{\gamma}, a, A\right) \\
& (0<\theta<\pi, \quad 0<r<R / 2) .
\end{aligned}
$$

Let $\left(r_{j}\right)$ be a sequence of Pólya peaks of order $\mu$ of $T(r, a, A)($ or $T(r, f))$ and $\left(r_{j^{\prime}}^{\prime}\right)$ be the sequence occuring in the definition of Pólya peaks (see $[1, p .418]$ ) such that $r_{j^{\prime}}^{\prime} / r_{j} \rightarrow \infty(j \rightarrow \infty)$.

Let us set

$$
s_{j}=\left(r_{j}\right)^{1 / \gamma} \text { and } s_{j^{\prime}}^{\prime}=\left(r_{j^{\prime}}^{\prime}\right)^{1 / \gamma}
$$

The following relations are valid:

$$
\begin{gathered}
\int_{0}^{s_{j}^{\prime}} N\left(t^{\gamma}, 0, F\right) P\left(t, s_{j}, \pi-\theta\right) d t \leqslant(1-\delta(a, f)) T\left(r_{j}, a, A\right) \\
\times\left\{\frac{\sin (\pi-\theta) \gamma \mu}{\sin \pi \gamma \mu}+o(1)\right\}, \\
\int_{0}^{s^{\prime} j^{\prime}} T\left(t^{\gamma}, a, A\right) P\left(t, s_{j}, \theta\right) d t \leqslant T\left(r_{j}, a, A\right)\left\{\frac{\sin \theta \mu \gamma}{\sin \pi \mu \gamma}+o(1)\right\}, \\
(j \rightarrow \infty, 0<\theta<\pi),
\end{gathered}
$$


where $o(1)$ does not depend on $\theta$,

$$
\frac{s_{j}}{s_{j^{\prime}}^{\prime}} T\left(\left(s_{j^{\prime}}^{\prime}\right)^{\gamma}, a, A\right)=o\left(T\left(r_{j}, a, A\right)\right), j \rightarrow \infty .
$$

Setting $r=s_{j}$ and $R=s_{j^{\prime}}^{\prime}$ in (2.5) and taking the relations (2.6), (2.7), (2.8) into account, we get $(j \rightarrow \infty, 0<\theta<\pi)$

$$
v\left(s_{j} e^{i \theta}\right) \leqslant T\left(r_{j}, a, A\right)\left\{\frac{\sin \theta \gamma \mu+(1-\delta(a, f)) \sin (\pi-\theta) \gamma \mu}{\sin \pi \gamma \mu}+o(1)\right\} .
$$

From the definition of $\gamma$ we have

$$
1-\delta(a, f)=\cos \pi \gamma \mu .
$$

We write the inequality (2.9) in the form

$$
\begin{aligned}
v\left(s_{j} e^{i \theta}\right) \leqslant & T\left(r_{j}, a, A\right)\left\{\cos (\pi-\theta) \gamma \mu+\alpha_{j}\right\} \\
& (j=1,2, \cdots, 0<\theta<\pi)
\end{aligned}
$$

where $\alpha_{j} \rightarrow 0$ as $j \rightarrow \infty$. Further, following [1, p.433-434], we arrive at the relation (2.3).

(2) $4 / \mu \arcsin \sqrt{(\delta(a, f) / 2)} \geqslant 2 \pi$.

In this case, we choose a number $d$ such that

and

$$
\begin{gathered}
0<d<\delta(a, f) \\
\frac{4}{\mu} \arcsin \sqrt{\frac{d}{2}}<2 \pi .
\end{gathered}
$$

Set

$$
\gamma=\frac{2}{\pi \mu} \arcsin \sqrt{\frac{d}{2}}
$$

by similar reasoning, we arrive at

$$
\sigma(a) \geqslant \frac{4}{\mu} \arcsin \sqrt{\frac{d}{2}} .
$$

Letting $d \uparrow d_{0}=2 \sin ^{2}(\mu \pi / 2)$, we obtain the desired result

$$
\sigma(a)=2 \pi
$$

Theorem 2.1 is proved. 
THEOREM 2.2. Let $f(z)$ be an $n$-valued algebroid function of lower order $\mu(0<\mu<\infty)$, defined by the equation (1.1), and $q \geqslant 2 \mu$ be an integer. If

$$
\delta(a, f) \geqslant 1-\cos \frac{\mu \pi}{q},
$$

then

$$
\sigma(a) \geqslant \frac{2 \pi}{q}
$$

Proof: The proof of this theorem is similar to the proof of case (1) in Theorem 2.1. Let us only observe that we must choose $\gamma=1 / q$ and apply inequality (2.10) to relation (2.9). Relation (4.16) from $[1$, p.433] reduces to the desired inequality (2.11). $]$

\section{Applications}

LEMmA 3.1. Let $f(z)$ be an $n$-valued algebroid function of lower order $\mu(0<\mu<\infty)$ and let $a_{i}(i=0,1, \cdots, n)$ be any $n+1$ distinct complex numbers. Choose $\Lambda(r)=(T(r, f))^{1 / 2}$ and define the sets $E_{\Lambda}\left(r, a_{j}\right)$ in $(-\pi, \pi]$ by

$$
E_{\Lambda}\left(r, a_{j}\right)=\left\{\theta: \frac{\|A(z)\| \cdot\left\|a_{j}\right\|}{\left|F\left(z, a_{j}\right)\right|}>e^{\Lambda(\gamma)}, z=e^{i \theta_{r}}\right\}(j=0,1, \cdots, n),
$$

Then there exists a positive number $r_{0}>0$ such that $r \geqslant r_{0}$

$$
\bigcap_{j=0}^{n} E_{\Lambda}\left(r, a_{j}\right)=\emptyset .
$$

Proof: We assume that $a_{j} \neq \infty(j=0,1, \cdots, n)$ without loss of generality. Suppose that

$$
E(r)=\bigcap_{j=0}^{n} E_{\Lambda}\left(r, a_{j}\right) \neq \emptyset .
$$

We choose $\theta_{0} \in E(r)$ and consider the following system of $n+1$ equations.

$$
F\left(r e^{i \theta_{0}}, a_{j}\right)=\sum_{k=0}^{n} A_{k}\left(r e^{i \theta_{0}}\right) a_{j}^{n-k} \quad(j=0,1, \cdots, n) .
$$

Since the determinant of the coefficients

$$
\operatorname{det}\left(a_{j}^{n}, a_{j}^{n-1}, \ldots, a_{j}, 1\right) \neq 0,
$$


we can solve this system for the unknowns $A_{j}\left(r e^{i \theta_{0}}\right)(0 \leqslant j \leqslant n)$ and obtain (for some constants $b_{j k}$ ):

$$
A_{k}\left(r e^{i \theta_{0}}\right)=\sum_{j=0}^{n} b_{j k} F\left(r e^{i \theta_{0}}, a_{j}\right), \quad(k=0,1, \cdots, n)
$$

so that

$$
\begin{aligned}
\left|A_{q}\left(r e^{i \theta_{0}}\right)\right| & =\max _{0 \leqslant k \leqslant n}\left|A_{k}\left(r e^{i \theta_{0}}\right)\right| \\
& \leqslant \max _{0 \leqslant k \leqslant n} \sum_{j=0}^{n}\left|b_{j k}\right| \cdot\left|F\left(r e^{i \theta_{0}}, a_{j}\right)\right| \\
& \leqslant C\left|F\left(r e^{i \theta_{0}}, a_{s}\right)\right|,(0 \leqslant s \leqslant n)
\end{aligned}
$$

where $C$ is a constant and

$$
\left|F\left(r e^{i \theta_{0}}, a_{s}\right)\right|=\max _{0 \leqslant j \leqslant n}\left|F\left(r e^{i \theta_{0}}, a_{j}\right)\right| .
$$

This means that for fixed $r$

$$
\begin{aligned}
\frac{\left\|A\left(r e^{i \theta_{0}}\right)\right\| \cdot\left\|a_{s}\right\|}{\left|F\left(r e^{i \theta_{0}}, a_{s}\right)\right|} & \leqslant \frac{(n+1)^{1 / 2}\left|A_{q}\left(r e^{i \theta_{0}}\right)\right| \cdot\left\|a_{s}\right\|}{\left|F\left(r e^{i \theta_{0}}, a_{s}\right)\right|} \\
& \leqslant(n+1)^{1 / 2} C\left\|a_{s}\right\|=\text { constant, }
\end{aligned}
$$

which for sufficiently large $r$ contradicts the assumption that $\theta_{0}$ belongs to $E_{\Lambda}\left(r, a_{s}\right)$. Lemma 3.1 is thus proved.

Lемма 3.2. Let $f(z)$ be an $n$-valued algebroid function of lower order $\mu(0<\mu<\infty)$, defined by the equation (1.1) and

$$
\Lambda(r)=(T(r, f))^{1 / 2}
$$

Then, on summing all the deficient values a of $f(z)$, we have

$$
\sum_{a} \sigma(a) \leqslant \sum_{a} \sigma_{\Lambda}(a) \leqslant 2 n \pi
$$

Proof: Let $a_{j}(j=1,2, \cdots, N)$ be any $N$ deficient values of $f(z)$. The sets $E_{\Lambda}\left(r, a_{j}\right)(1 \leqslant j \leqslant N)$ are defined by (3.1). Since for each $\theta_{0} \in(-\pi, \pi], \theta_{0}$ can belong to at most $n$ of the sets $E_{\Lambda}\left(r, a_{j}\right)(1 \leqslant j \leqslant N)$ for sufficiently large $r$,

$$
\sum_{k=1}^{N} \sigma\left(a_{k}\right) \leqslant \sum_{k=1}^{N} \sigma_{\Lambda}\left(a_{k}\right)=\sum_{k=1}^{N} \lim _{j \rightarrow \infty} \text { meas } E_{\Lambda}\left(r_{j}, a_{k}\right) \leqslant 2 n \pi .
$$


Since $N$ can be arbitrarily large, Lemma 3.2 is thus proved.

THEOREM 3.1. Let $f(z)$ be an $n$-valued algebroid function of lower order $\mu(0<\mu<\infty)$, defined by the equation (1.1) and $q(\geqslant 2 \mu)$ be an integer. If $f(z)$ has more than $n q$ deficient values, then there are at most $n q-1$ deficient values $a_{k}(k=1,2, \cdots, n q-1)$ such that

$$
\delta_{k}=\delta\left(a_{k}, f\right) \geqslant 1-\cos \frac{\mu \pi}{q},(k=1,2, \cdots, n q-1) .
$$

Proof: Assume that the assertion is false; we choose $n q+1$ distinct deficient values $a_{k}(k=1,2, \cdots, n q+1)$ of $f(z)$ such that

$$
\begin{aligned}
& \delta_{1} \geqslant \delta_{2} \geqslant \cdots \delta_{n q} \geqslant 1-\cos \frac{\mu \pi}{q}, \\
& \delta_{n q+1}>0,\left(q \geqslant 2 \mu, \delta_{k}=\delta\left(a_{k}, f\right), 1 \leqslant k \leqslant n q+1\right) .
\end{aligned}
$$

Choosing the integer $s(\geqslant n q)$ large enough, (3.3) implies

$$
\delta_{n q+1} \geqslant 1-\cos \frac{\mu \pi}{s} \text {. }
$$

Now let $\left(r_{j}\right)$ be a sequence of Pólya peaks of $T(r, f)$ and let

$$
\Lambda(r)=(T(r, f))^{1 / 2}
$$

by Theorem $2.2,(3.2)$ and (3.4) imply

So

$$
\begin{gathered}
\sigma\left(a_{k}\right) \geqslant \frac{2 \pi}{q}, \quad \sigma\left(a_{n q+1}\right) \geqslant \frac{2 \pi}{s}, \quad k=1,2, \cdots, n q . \\
\sum_{k=1}^{n q+1} \sigma_{\Lambda}\left(a_{k}\right) \geqslant \sum_{k=1}^{n q+1} \sigma\left(a_{k}\right) \geqslant 2 n \pi+\frac{2 \pi}{s} .
\end{gathered}
$$

This contradicts Lemma 3.2, so Theorem 3.1 is proved.

THEOREM 3.2. Let $f(z)$ be an n-valued algebroid function of lower order $\mu(0 \leqslant \mu \leqslant \infty)$, defined by the equation (1.1). Then on summing over all the deficient values $a$ of $f(z)$, we have

$$
\sum_{a} \sqrt{\delta(a, f)} \leqslant n(\sqrt{2} \mu \pi+2 \mu+1) .
$$

Proof: We consider the following two cases.

(1) If $\mu=0$, by a result of $\mathrm{Gu}([4]), f(z)$ has at most $n$ deficient values, so that Theorem 3.2 holds. 
(2) If $0<\mu<\infty$, we assume that

$$
a_{1}, a_{2}, \cdots, a_{n}, \cdots
$$

are all the deficient values of $f(z)$ and assume that (3.5) has been ordered so that

$$
\delta_{1} \geqslant \delta_{2} \geqslant \cdots \geqslant \delta_{n} \geqslant \cdots,
$$

where $\delta_{k}=\delta\left(a_{k}, f\right), k=1,2, \cdots, n, \cdots$.

Let $q=[2 \mu]+1$ and $m$ be an integer. If $m \leqslant n q$, it is trivial that

$$
\sum_{i=1}^{m} \sqrt{\delta\left(a_{i}, f\right)} \leqslant n q \leqslant n(2 \mu+1) .
$$

If $m>n q$, by Theorem 3.1 we have

$$
\delta_{n q}<1-\cos \frac{\mu \pi}{q} .
$$

Hence, with each $\delta_{n q+i}>0(1 \leqslant i \leqslant m-n q)$, we may associate a positive integer $q_{i}$ such that

$$
1-\cos \frac{\mu \pi}{q_{i}+1} \leqslant \delta_{n q+i}<1-\cos \frac{\mu \pi}{q_{i}}, i \geqslant 1 .
$$

By Lemma 3.2 and Theorem 2.2, we get

$$
\sum_{i=1}^{m-n q} \frac{2 \pi}{q_{i}+1} \leqslant \sum_{i=n q+1}^{m} \sigma\left(a_{i}\right) \leqslant 2 n \pi
$$

so that

$$
\sum_{i=1}^{m-n q} \frac{1}{q_{i}} \leqslant \sum_{i=1}^{m-n q} \frac{2}{q_{i}+1} \leqslant 2 n .
$$

From the second inequality in (3.6), we deduce

$$
\delta_{i}^{1 / 2}<\sqrt{2} \sin \frac{\mu \pi}{2 q_{i}}<\frac{\pi \mu}{q_{i} \sqrt{2}}
$$

and hence

$$
\sum_{i=n q+1}^{m} \sqrt{\delta\left(a_{i}, f\right)} \leqslant \sum_{i=1}^{m-n q} \frac{\mu \pi}{\sqrt{2} q_{i}} \leqslant \sqrt{2} n \mu \pi \text {. }
$$

Therefore

$$
\sum_{i=1}^{m} \sqrt{\delta\left(a_{i}, f\right)} \leqslant \sqrt{2} n \mu \pi+n q \leqslant n(\sqrt{2} \mu \pi+2 \mu+1) .
$$

Since $m$ can be arbitrarily large, Theorem 3.2 follows from (3.8). 


\section{REFERENCES}

[1] A. Baernstein, 'Proof of Edrei's spread conjecture', Proc. London Math. Soc. 26 (1973), 418-434.

[2] A. Baernstein, 'Integral means, univalent functions and circular symmetrizations', Acta Math. 133 (1974), 139-169.

[3] A. Goldberg, 'Certain questions of the theory of value distribution', in Recent Investigations on Single-Valued Analytic Functions, (Russian translation), Editor G. Wittich, pp. 263-300 (Fizmatgiz, Moscow, 1960).

[4] Yongxing $\mathrm{Gu}$, 'The growth of algebroid functions with several deficient values', Contemp. Math. 25 (1983), 45-49.

[5] K. Niino, 'Spread relation and value distribution in an angular domain of holomorphic curves', Kodai Math. Sem. Rep. 26 (1977), 361-371.

[6] G. Valiron, 'Sur la dérivee des fonctions algébroides', Bull. Soc. Math. 59 (1931), 17-39.

Department of Mathematics

Shandong University

Jinan, Shandong 250100

Peoples Republic of China 